\title{
ОБ АНАЛИТИЧЕСКОЙ ЗАПИСИ ГРАФОВ КОНЕЧНЫХ
} ABTOMATOB

При практической работе с направленными графами асинхронных конечных автоматов возникает необходимость допущения одновременного выхода нескольких одинаково отмеченных дуг из одного и того же узла (события). Это допущение не вносит существенных изменений в уже существующую математическую теорию конечных автоматов [1, 2 и др.], так как путем перекодирования всегда возможно объединить все одинаково отмеченные дуги. Оно важно для облегчения аналитической записі практических схем с параллельно работающими узлами, ибо вносит инвариантность записи одной ветви относительно изменений в других ветвях.

При допущении разбиения события на несколько одновременно происходящих подсобытий для аналитической записи автомата невозможно удобно использовать двухмерные таблицы состояний и выходов [1], так как это допущение требует применения многомерных таблиц. Предлагаемый линейный метод записи позволяет обойти это затрудненце. Дополнительным удобством предлагаемого метода является относительная легкость ввода получаемой записи в современные ЭЦВМ, максимально приспособленные для линейных записей.

В графе автомата, допускающего параллельную работу ветвей (параллельные потоки информации), можно выделить три основных типа узлов:

1. Узлы, входным сигналом для которых служит асинхронный сигнал скончания события, являющегося предшествующим для данного события. Из узлов типа 1 выходит точно одна дуга. Имеются две разновидности:

la. В узел не входит ни одной или только одна дуга. Это начальныє или текущие линейные узлы, в которых не происходит увеличения или уменьшения количества параллельных ветвей графа автомата.

1б. В узел входит более одной дуги. Такие узлы являются стягивающими и в них количество параллельных ветвей автомата уменьшается.

2. Узлы, входным сигналом для которых, кроме тактирующего (разрешающего) сигнала об окончании предшествовавшего события, служат также так наз. внешние сигналы $\left.{ }^{3,}{ }^{4}\right]$. Множество входных признаков, об разуюшее сигнал, может быть разделено на взаимно пересекающиеся подмножества, отмечающие разные выходящие из данного узла дугю. Узлы типа 2 могут быть подразделены на два подтипа:

2а. В узел не входит ни одной или только одна дуга. Если из узла выходит точно одна дуга, то такой узел может задержать начало следующего по дуге события до момента появления буквы, отмечающей эту дугу. Следовательно, узел типа 2а с одной выходящей дугой как бы имеет цикл единичной длины, отмеченный дополнением отмечающей эту дугу буквы и генерирующий пустую выходную букву. Если из узла типа 2 a выходит более одной дуги, то в случае одновременного наличия отмечающих букв для более чем одной дуги все эти переходы совершатся одновременно и количество активных (т. е. работающих) ветвей увеличится. Если на входе не найдется отмечающсй буквы хотя бы для одной из дуг, выходящих из данного узла, то этот узел становится концевым узлом данного случая работы автомата по этой ветви.

2б. В узел входит более одной дуги. Узел является стягивающим. Относительно выходящих дуг выполняются точно те же условия, что и для типа $2 \mathrm{a}$. 
3. Концевой узел, из которого не выходит ни одной дуги. По типу входов может одновременно быть и стягиваюгци.

Алгоритм аналитической записи графа основывается на вышеприведенной классификации узлов и состоит из 5 шагов.

1. Все узлы графа - события - обозначают взаимно различными идентификаторами или порядковыми номерами.

2. Всем участкам, состоящим из одних только линейных операторов типа 1а, присваивают идентификаторы или порядковые номера, отличные от присвоенных при шаге 1. В такой линейный участок включается также следующий за узлами типа 1 узел типа 2 или 3. Этим обеспечивается указание связей в графе.

3. Если два или больше линейных участка, сведенных по шагу 2 , были до этого разделены только узлами типа 1б, то подобную последовательность обозначают идентификатором или номером, отличным от ранее введенных. Узел 16 включается в следующий за ним участок. Шаги 2 и 3 невозможно объединить в один, так как нужна метка для перехода.

4. Для каждого из узлов типа 2 записывают список условий в виде последовательности пар «<входная буква >, <оператор, следующий за >», разделенных точкой с запятой. В качестве входной буквы может быть записано произвольное логическое условие в принятом для записи алфавите. В качестве «оператора, следующего за» может быть использован оператор перехода АЛГОЛа [6], просто идентификатор или номер следующего оператора, или любая другая однозначно определенная запись оператора перехода.

5. Конец аналитической записи.

Полученное отображение графа имеет однозначное обратное отображение в граф, изоморфный первоначальному.

Предлагаемый метод был применен в несколько измененном виде для записи и моделирования работы цифровых схем [5], а также для записи произвольных конечных автоматов из ['] и графов сетевого графика.

\section{Л И ТЕ Р А Т У Р А}

1. Гл ушков В. М., Синтея цифровых автоматов, М., 1962.

2. К об ри н ски й Н. Е., Т р а хтен брот Б. А., Введение в теорию конечных автоматов, М., 1962.

3. Г л уш к о в В. М., Изв. АН СССР. Сер. техн. киберн., № 1, 3-8 (1964).

4. М а ц е в и т ы й Л. В., Изв. АН СССР. Сер. техн. киберн., № 1, 9-19 (1964).

5. С а лу м Х. Л., Изв. АН ЭССР. Сер. физ.-матем. и техн. наук, 14, № 3, 464-4i2 (1965).

6. Аг е в М. И., Основы алгоритмического языка АЛГОЛ-60, М., 1964.
Институт кибернетики
Академии наук Эстонской ССР
Поступила в редакцию 13/I 1966

\section{H. SALUM}

\section{LOPLIKE AUTOMAATIDE GRAAFIDE ANALUUTILISEST KIRJELDAMISEST}

Artiklis põhjendatakse selliste löplike määratud automaatide vajalikkus, mille graafides väljub samast sõlmest mitu ühtemoodi märgitud kaart. Esitatakse lühike sõlmede klassifikatsioon ja graafi lineaarse üleskirjutamise algoritm.

\section{H. SALUM}

\section{ON THE ANALYTICAL DESCRIPTION OF THE GRAPHS OF THE FINITE AUTOMATA}

The author discusses the necessity for finite-state determined automata, the graphs of which issue several identically labelled arcs from one and the same vertex.

A short classification of the vertices and an algorithm for the linear description of the graph are given. 